\title{
Calprotectina fecal como marcador diferencial entre patología gastrointestinal orgánica y funcional
}

\author{
A. Bonnín Tomàs, M. Vila Vidal y A. Rosell Camps ${ }^{1}$ \\ Sección de Bioquímica. Servicio de Análisis Clínicos. ${ }^{1}$ Unidad de Gastroenterología. Servicio de Pediatría. Hospital \\ Universitario Son Dureta. Palma de Mallorca
}

\section{RESUMEN}

Introducción: la determinación de calprotectina en heces se está afianzando en los últimos años como un marcador no invasivo para el diagnóstico diferencial entre patología gastrointestinal orgánica y funcional. Su uso es útil sobre todo en niños que requieren anestesia general para una colonoscopia. El objetivo de este estudio es evaluar la sensibilidad y utilidad de la calprotectina fecal (CPF) en pacientes pediátricos con signos y síntomas sugestivos de enfermedad inflamatoria intestinal (EII) con el fin de evitar técnicas invasivas innecesarias y poder discriminar entre patología gastrointestinal orgánica y funcional.

Material y métodos: se determinó la concentración de calprotectina mediante enzimoinmunoanálisis en una única muestra de heces de 47 niños (edad media: 10,1 años) con algún síntoma de patología gastrointestinal sugestivo de organicidad. Trece niños fueron diagnosticados de patología funcional y 34 de patología orgánica. Entre estos, 15 con EII y el resto con patologías orgánicas de distinto origen (no-EII). Se incluyeron 13 niños sanos como controles.

Resultados: el grupo de niños con EII presentó valores de CPF [mediana (rango interquartil); $1.219 \mu \mathrm{g} / \mathrm{g}(322-2.967)]$ significativamente más altos que el grupo con patología gastrointestinal funcional [20 $\mu \mathrm{g} / \mathrm{g}(16-25) ; \mathrm{p}<0,0001$ ], el grupo con patología orgánica no-EII [113 $\mu \mathrm{g} / \mathrm{g}(36-193) ; \mathrm{p}=0,002$ ] y el control [25 $\mu \mathrm{g} / \mathrm{g}(19-32) ; \mathrm{p}<0,0001]$. Las concentraciones también fueron más altas en el grupo de niños con patología orgánica no-EII respecto al grupo con patología funcional $(p=0,002)$ y al control $(p=0,004)$. No hubo diferencias entre el grupo control y los niños con patología funcional $(\mathrm{p}=0,264)$.

Discusión: la CPF es un marcador sensible, pero no específi$\mathrm{co}$, que permite seleccionar pacientes con EII, que requieren colonoscopia para el diagnóstico definitivo y evitar así pruebas invasivas a pacientes con patología gastrointestinal funcional.

Palabras clave: Calprotectina fecal. Enfermedad inflamatoria intestinal. Patología gastrointestinal funcional. Pediatría.

\begin{abstract}
Introduction: there is growing evidence showing the importance of the fecal calprotectin assay in differentiating organic from functional gastrointestinal disease. It is a simple, non-invasive biomarker that is especially useful in children, who may require general anesthesia for colonoscopy. The aim of this study was to assess the use and sensitivity of fecal calprotectin (FCP) in pediatric patients with signs and symptoms of IBD to avoid unnecessary invasive techniques and to distinguish between organic and functional gastrointestinal pathology.
\end{abstract}

Material and methods: a single stool sample was collected from 47 children (mean age: 10.1 years) referred for non-specific gastrointestinal symptoms suggestive of organicity. On the basis of clinical criteria 13 children had functional bowel disorders and 34 had organic gastrointestinal disease, 15 with IBD and 19 with other organic (non-IBD) gastrointestinal conditions. Thirty healthy children were included as controls. Calprotectin concentrations were measured by enzyme immunoassay.

Results: children with IBD had FCP levels [median (interquartile range); $1,219 \mu \mathrm{g} / \mathrm{g}(322-2,967 \mu \mathrm{g} / \mathrm{g})]$ higher than children with functional gastrointestinal disease $[20 \mu \mathrm{g} / \mathrm{g}(16-25 \mu \mathrm{g} / \mathrm{g}) ; \mathrm{p}<$ 0.0001 , those with organic non-IBD disease [113 $\mu \mathrm{g} / \mathrm{g}(36-193$ $\mu \mathrm{g} / \mathrm{g}) ; \mathrm{p}=0.002]$, and healthy children [25 $\mu \mathrm{g} / \mathrm{g}(19.2-32.5 \mu \mathrm{g} / \mathrm{g})$; $p<0.0001$. Fecal calprotectin concentration also was significantly higher in children with organic (non-IBD) disease as compared to controls $(p=0.004)$ and children with functional pathology $(p=$ 0.002). FCP levels were similar in controls and children with functional gastrointestinal disease $(p=0.264)$.

Discussion: $\mathrm{CPF}$ is a sensitive, but not disease-specific, marker to identify patients with IBD who should undergo diagnostic colonoscopy, and to avoid unnecessary invasive procedures in patients with functional gastrointestinal disorders.

Key words: Fecal calprotectin. Inflammatory bowel disease. Functional gastrointestinal diseases. Pediatry.

Bonnín Tomàs A, Vila Vidal M, Rosell Camps A. Calprotectina fecal como marcador diferencial entre patología gastrointestinal orgánica y funcional. Rev Esp Enferm Dig 2007; 99: 689-693. 


\section{INTRODUCCIÓN}

El dolor abdominal funcional es una causa frecuente de asistencia en las consultas de atención primaria y de gastroenterología, requiriendo incluso en algunas ocasiones ingresos hospitalarios. El principal reto es diferenciarlo de una patología orgánica que a veces puede conllevar la realización de múltiples pruebas diagnósticas con los riesgos que estas pueden acarrear, sus efectos secundarios y las pérdidas de horas escolares para los pacientes pediátricos y laborales para los padres.

Muchos síntomas clínicos presentes en el dolor abdominal funcional son comunes con otros trastornos orgánicos, en particular con los de la enfermedad inflamatoria intestinal (EII) (1). Ante la sospecha de EII se aconseja realizar una gammagrafía de leucocitos marcados y una colonoscopia con toma de biopsia para confirmar el diagnóstico y determinar el grado de inflamación. Son técnicas invasivas con un riesgo inherente de complicaciones e irradiación para el paciente y que en el caso de la colonoscopia en pacientes pediátricos requiere de anestesia general. Los parámetros bioquímicos de inflamación usados clásicamente, como la velocidad de sedimentación globular y la proteína $\mathrm{C}$ reactiva, carecen de suficiente especificidad o sensibilidad diagnóstica (2). La determinación de calprotectina en heces se está afianzando en los últimos años como un nuevo marcador útil de patología inflamatoria intestinal (3-7). Varios estudios demuestran, además, que existe asociación entre los niveles de calprotectina y el grado de inflamación, en consecuencia puede usarse para monitorizar la respuesta al tratamiento y predecir el riesgo de recidivas (8-12).

La calprotectina es una proteína fijadora de calcio y zinc que pertenece a la familia de proteínas S-100. Su estructura molecular consiste en un heterotrímero $36,5 \mathrm{kD}$ formada por 2 cadenas pesadas y 1 ligera no glicosiladas. Esta proteína constituye aproximadamente el $60 \%$ de las proteínas solubles del citosol de neutrófilos y también es muy abundante en monocitos y macrófagos (13).

Aunque su función biológica no está completamente dilucidada, podría tener un papel importante en la regulación de la inflamación y procesos neoplásicos. Parece que ejerce una función protectora en procesos inflamatorios e infecciosos y también presenta actividad antiproliferativa, sin embargo un incremento excesivo podría inducir daño celular (14). Se ha medido su concentración en plasma y otros fluidos biológicos (saliva, LCR, sinovial) y en heces, donde se halla más concentrada que en el resto. Se encuentra elevada en múltiples estados patológicos tanto inflamatorios (fibrosis quística, artritis reumatoide, enfermedad de Crohn, colitis ulcerosa e infecciones bacterianas) como neoplásicos (cáncer colorrectal) (15).

A nivel del tracto gastrointestinal, patologías inflamatorias con distinta etiología provocan un aumento de la permeabilidad de la mucosa que induce la migración de granulocitos y monocitos hacia el lumen intestinal (15).
La subsiguiente activación y muerte de estas células libera gran cantidad de calprotectina (16), que es excretada en las heces. La determinación de calprotectina en heces, presenta buena correlación con la excreción fecal de leucocitos marcados con Indio111, considerada como técnica de referencia para medir la actividad inflamatoria del intestino (9).

Cuando la calprotectina se encuentra unida a calcio presenta una elevadísima resistencia al calor y a la degradación metabólica ejercida por enzimas bacterianas y por las propias proteasas intestinales $(8,17)$. Estas propiedades permiten que se elimine intacta por las heces y le confieren ventaja como marcador bioquímico, no invasivo, para el cribaje de inflamación intestinal frente a otros marcadores utilizados (lactoferrina, elastasa neutrofílica o estearasa leucocitaria).

Actualmente está disponible un ensayo mejorado para la determinación de calprotectina mediante ELISA (18) que, como principales ventajas respecto al original, requiere menor cantidad de muestra y aumenta el rendimiento de la solución de extracción. Se trata de una técnica simple, sensible, exacta, reproducible y más económica.

Se han realizado varios estudios en adultos y niños con patología gastrointestinal, pero con frecuencia los resultados no son comparables dado el uso de las distintas metodologías con distintas unidades y sensibilidades. Con el método ELISA original se consideraba un límite superior de referencia de $10 \mathrm{mg} / \mathrm{l}$ mientras que con el método mejorado se establece el límite en $50 \mu \mathrm{g} / \mathrm{g}$ de heces.

El objetivo de este estudio es evaluar la sensibilidad y utilidad de la calprotectina fecal (CPF) en pacientes pediátricos con signos y síntomas sugestivos de enfermedad inflamatoria intestinal con el fin de evitar técnicas invasivas innecesarias y poder discriminar entre patología gastrointestinal orgánica y patología gastrointestinal funcional.

\section{MATERIAL Y MÉTODOS}

Estudio retrospectivo durante el periodo 2003-2005, de 47 pacientes con algún síntoma de patología gastrointestinal, que habían sido remitidos a la unidad de gastroenterología pediátrica del Hospital Universitario Son Dureta (Palma de Mallorca) y a los que en alguna visita se les había solicitado calprotectina fecal por presentar signos y síntomas sugestivos de patología orgánica (dolor abdominal intenso e invalidante, diarrea crónica, estancamiento ponderal, rectorragia). La edad media de los niños era de 10,1 años, con edades comprendidas entre 3 meses y 15,3 años. Siguiendo criterios clínicos, pruebas de laboratorio, de imagen y endoscópicas y en relación a su evolución cumpliendo los criterios de patología funcional establecidos en la reunión de Roma II (19), trece niños fueron diagnosticados de patología funcional y treinta y cuatro de patología orgánica. Entre este último grupo, 15 presentaban enfermedad inflamatoria intestinal (EII), de ellos 3 colitis ulcerosa (CU) y 12 enfermedad de Crohn 
(EC), y el resto presentaban patologías orgánicas de distinto origen (colitis infecciosas, alergias alimentarias, etc.). Se incluyeron como controles 13 niños sanos, familiares del personal del laboratorio, que accedieron al estudio tras consentimiento informado.

Se recogió una única muestra de heces por paciente en recipientes de plástico y en menos de 48 horas se remitieron al laboratorio, donde se congelaron a $-70{ }^{\circ} \mathrm{C}$ hasta su análisis. La calprotectina es estable en heces a temperatura ambiente al menos durante 72 horas y más de 6 meses congelada. No es necesario homogenizar la muestra ya que no presenta variabilidad entre distintas tomas de la misma (18). Las muestras se prepararon y analizaron de acuerdo con las instrucciones del fabricante (Calprest, Eurospital, Trieste, Italia).

Las concentraciones de calprotectina se calcularon a partir de la curva de regresión polinomial de cuarto orden, obtenida con los estándares del kit $(6,25 ; 12,5 ; 25$; 50 y $100 \mathrm{ng} / \mathrm{ml}$ ). Los resultados se expresan en $\mu \mathrm{g} / \mathrm{g}$. Las muestras cuyos valores superaron la linealidad de la técnica, con lectura de absorbancia superior a la del calibrador de mayor concentración, fueron diluidas como indica el fabricante: con el sobrenadante obtenido a partir de los extractos se realizaron diluciones crecientes 1:100, 1:200 hasta un límite de 1:500 veces. La calprotectina también es estable en los extractos congelados y descongelados hasta 4 veces (18). El límite inferior de detección del ensayo es $15,6 \mu \mathrm{g} / \mathrm{g}$.

El análisis de los datos se realizó con el paquete estadístico SPSS v.11. El test de Kolmogorov-Smirnov mostró que los valores de concentración de calprotectina no seguían una distribución normal. En consecuencia, los resultados se expresan en mediana y rango intercuartil. Los valores entre los distintos grupos se compararon con la prueba estadística U de Mann-Whitney. Asumimos una diferencia estadísticamente significativa cuando $\mathrm{p}<0,05$.

\section{RESULTADOS}

Los resultados obtenidos de la concentración de CPF en los distintos grupos se muestran en la tabla I. No se incluyen en esta tabla, 2 pacientes con EC, un paciente con CU y otro niño con alergias múltiples, cuyos valores de

Tabla I. Valores de mediana, rango y percentil 95 de calprotectina fecal $(\mu \mathrm{g} / \mathrm{g})$ en pacientes con enfermedad inflamatoria intestinal (EII), patología gastrointestinal orgánica (no ElI), patología gastrointestinal funcional y en el grupo control

\begin{tabular}{lcccc}
\hline & Ell & $\begin{array}{c}\text { Orgánica } \\
\text { (no Ell) }\end{array}$ & Funcional & Control \\
\hline $\mathrm{N}$ & 12 & 18 & 13 & 13 \\
Mediana & $1.218,9$ & 112,6 & 20,0 & 25,0 \\
Rango intercuartil & $321,8-2,966,8$ & $36,5-193,1$ & $15,6-25,4$ & $19,2-32,5$ \\
Percentil 95 & 3797,0 & 4011,1 & 137,5 & 61,3 \\
\hline
\end{tabular}

calprotectina superaron la linealidad de la técnica, con concentraciones $>250 \mu \mathrm{g} / \mathrm{g}$, pero no pudimos realizar diluciones mayores como indicamos en la metodología.

El grupo de niños con EII presentó valores de CPF significativamente más altos que el grupo de niños con patología gastrointestinal de tipo funcional $(\mathrm{p}<0,0001)$, el grupo con patología orgánica no EII $(\mathrm{p}=0,002)$ y el control $(\mathrm{p}<0,0001)$. Las concentraciones también fueron significativamente más altas en el grupo de niños con otro tipo de patología orgánica (no EII) respecto al grupo con patología funcional $(\mathrm{p}=0,002)$ y al control $(\mathrm{p}=$ $0,004)$. En cambio no hubo diferencias en las concentraciones de calprotectina entre los niños del grupo control y los niños con patología gastrointestinal de origen funcional $(\mathrm{p}=0,264)$.

En la figura 1 está representada la distribución de los valores de calprotectina en cada uno de los grupos. Ningún niño diagnosticado de EII presentó valores de CPF inferiores a $50 \mu \mathrm{g} / \mathrm{g}$, el valor más bajo fue de $91,9 \mu \mathrm{g} / \mathrm{g}$ en un niño con EC. Entre el grupo con patología de tipo funcional tan solo un niño con dolor abdominal recurrente tuvo un nivel de calprotectina de $137,5 \mu \mathrm{g} / \mathrm{g}$ y en el resto fue $<50 \mu \mathrm{g} / \mathrm{g}$. Los niveles de calprotectina fueron $>50 \mu \mathrm{g} / \mathrm{g}$ en 13 de los 19 niños $(68,4 \%)$ con patología orgánica de etiología distinta a la EII. Dentro de este grupo encontramos niveles $>200 \mu \mathrm{g} / \mathrm{g}$ en 4 niños $(21,0 \%)$ : uno con alergias a múltiples alimentos (> $250 \mu \mathrm{g} / \mathrm{g})$, otro con enfermedad celiaca y diarrea crónica por transgresiones dietéticas $(354,7 \mu \mathrm{g} / \mathrm{g})$, uno con rectorragia y colonoscopia normal con probable fisura anal $(1.264,2 \mu \mathrm{g} / \mathrm{g})$ y en un niño con síndrome IPEX $(4011,1 \mu \mathrm{g} / \mathrm{g})$ que, de todos los pacientes, presentó el valor más alto de calprotectina. Con valores entre 50-200 $\mu \mathrm{g} / \mathrm{g}$ se incluyeron dos niños con gastritis por Helicobacter pylori, dos con alergia a proteínas de leche de vaca y a otros alimentos, uno con intolerancia a la lactosa, uno con litiasis biliar, un niño con enfermedad de

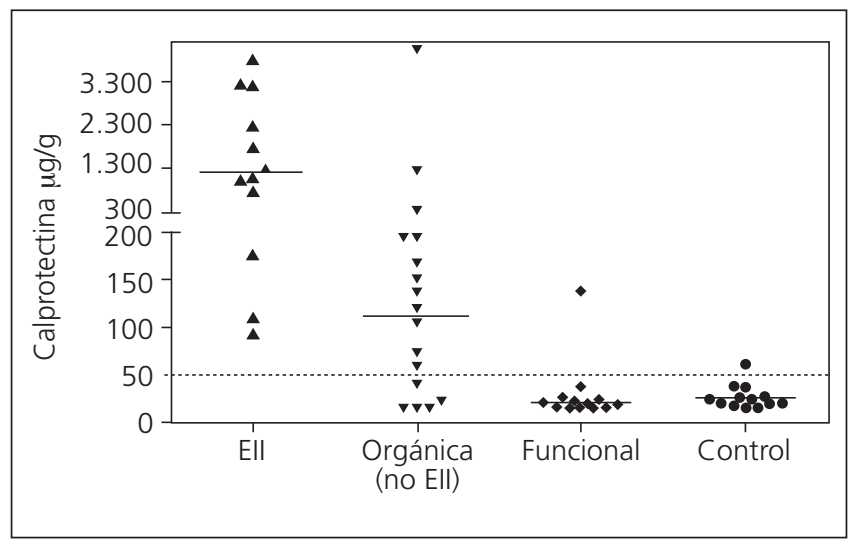

Fig. 1. Concentración de calprotectina fecal en niños con efermedad inflamatoria intestinal (EII) ( $n=12)$, patología gastrointestinal orgánica no Ell ( $n=18)$, patología gastrointestinal funcional $(n=13)$ y en el grupo control $(n=13)$. La línea sólida representa la mediana de cada grupo. La línea punteada corresponde a $50 \mu \mathrm{g} / \mathrm{g}$, considerado el límite superior de normalidad. 
Hirschprung, uno con síndrome de Wiscott-Aldrich y diarrea crónica, y uno con gastritis idiopática. En el grupo con EII, los niveles de calprotectina fueron $>200 \mu \mathrm{g} / \mathrm{g}$ en $12 \mathrm{de}$ los 15 niños estudiados (80\%).

\section{DISCUSIÓN}

En la práctica médica general existe la necesidad de una prueba simple que ayude a seleccionar aquellos pacientes con diarrea crónica o dolor abdominal crónico que precisen pruebas diagnósticas más complejas. La colonoscopia es el método de referencia para el diagnóstico de enfermedad inflamatoria intestinal pero se trata de una técnica cara, invasiva y no puede repetirse con frecuencia. También existe la video-enteroscopia en caso de sospechar EII con una colonoscopia normal, pero es otra prueba invasiva, sin experiencia en niños y no disponible en todos los centros (20). El uso de un marcador no invasivo, a la vez que fiable, sencillo y que se pueda repetir, es especialmente importante en los niños (21), ya que requieren anestesia general para realizar una colonoscopia (4).

Nuestro trabajo apoya resultados de estudios previos en niños y adultos $(5,18,22)$, que muestran como una única determinación de CPF puede ayudar a los gastroenterólogos en el diagnóstico diferencial entre la EII y otros síntomas gastrointestinales de etiología funcional. Tomando como límite superior de referencia $50 \mu \mathrm{g} / \mathrm{g}$, nuestros datos confieren una sensibilidad del $100 \%$ y una especificidad del $92 \%$ para discriminar entre EII y patología gastrointestinal funcional. El único niño con dolor abdominal funcional y $137 \mu \mathrm{g} / \mathrm{g}$ de CPF normalizó la concentración en una segunda determinación $(15,6 \mu \mathrm{g} / \mathrm{g})$, no incluida en el estudio, pasados 4 meses. En cambio pacientes con EII confirmada en los que se repitió la $\mathrm{CPF}$, cuyos valores tampoco fueron incluidos, mantenían niveles elevados. Sin embargo, para optimizar la eficacia diagnóstica de la prueba, algunos estudios postulan como límite superior de referencia concentraciones de calprotectina próximas a $100 \mu \mathrm{g} / \mathrm{g}(6)$. Un estudio reciente muestra un punto de corte óptimo de $217 \mu \mathrm{g} / \mathrm{g}$ con el fin de discriminar a pacientes adultos con enfermedad orgánica en colon $(4,23)$. La casa comercial establece como margen dudoso valores comprendidos entre 50 y $100 \mu \mathrm{g} / \mathrm{g}$. Si consideramos que la principal utilidad de la prueba es seleccionar a los pacientes que requieren pruebas diagnósticas invasivas, el uso de un punto de corte más bajo confiere mayor sensibilidad diagnóstica para EII aunque disminuya su especificidad.

El valor de referencia en niños sanos entre 4 y 17 años (24) se ha establecido por debajo de $50 \mu \mathrm{g} / \mathrm{g}$ de acuerdo con el límite indicado en el ensayo. Sin embargo, otros estudios muestran valores de calprotectina superiores a este límite en niños sanos del grupo control $(6,25)$. En nuestro grupo control un niño de siete años presentó una concentración de calprotectina de $61,3 \mu \mathrm{g} / \mathrm{g}$. Por otro lado, se han propuesto distintos valores de referencia de calprotectina fecal en recién nacidos. Durante los primeros días de vida, con una permeabilidad intestinal incrementada, los valores de calprotectina son más elevados $(25,26)$ que en niños y adultos. El hecho de presentar mayor permeabilidad intestinal se relaciona con una mayor migración transepitelial de neutrófilos, como se observa en adultos con EII.

A pesar de que el estudio se ha realizado con un reducido número de pacientes, los resultados confieren a la determinación de CPF utilidad como prueba candidata para seleccionar pacientes con EII, aunque desde el inicio de su aplicación ha demostrado ser inespecífica para este tipo de patología orgánica intestinal. Además de la EII, patologías de muy distinta etiología tales como infecciones, alergias a alimentos, fibrosis quística, neoplasias, etc., o terapia con antiinflamatorios no esteroideos, pueden dar resultados de calprotectina elevados $(4,14)$. En nuestra serie de datos, las concentraciones de calprotectina fueron significativamente más altas en el grupo de niños con EII en comparación al grupo con otro tipo de patología orgánica. Aún así, nuestros resultados tampoco permiten establecer valores de calprotectina que discriminen entre ambos grupos de patología. La CPF podría servir como un primer eslabón, cuando las manifestaciones clínicas no lo permiten, para diferenciar pacientes con posible patología gastrointestinal orgánica y pasar a un segundo nivel con exploraciones más caras, complejas e invasivas.

La gammagrafía con leucocitos marcados con isótopos radioactivos es la técnica de referencia para medir la actividad inflamatoria intestinal, pero es cara, emite radiaciones y no está disponible en todos los centros (27). Los niveles de CPF presentan buena correlación con esta técnica de referencia y por tanto, con el grado de inflamación intestinal, por ello puede usarse para monitorizar la respuesta al tratamiento $(8,9)$. No obstante, los niveles de calprotectina superan a menudo el límite de linealidad de la técnica, lo que implica tener que realizar varias diluciones, que además de encarecer mucho la determinación, retrasa la obtención del resultado y el diagnóstico. La casa comercial plantea la utilidad de la calprotectina solo como una prueba de cribado para el diagnóstico de EII. Recientemente se ha publicado un nuevo ensayo inmunofluorométrico que soluciona esta limitación de la técnica, ya que amplia hasta cuatro veces más el rango de linealidad del ELISA. Con ello se consigue reducir el número de muestras con concentraciones superiores al rango de medida de un 30 al $4 \%$ (28).

En conclusión, consideramos que la CPF, es una técnica rápida, fiable y reproducible, que permite seleccionar a pacientes con sintomatología gastrointestinal inespecífica que deben someterse a técnicas invasivas y más costosas y por tanto puede usarse como marcador bioquímico de rutina, no invasivo, para el diagnóstico y el seguimiento de pacientes con EII así como técnica de 
elección en el diagnóstico diferencial entre la patología gastrointestinal funcional y la de causa orgánica.

A pesar de todo, son necesarios estudios más completos con un mayor número de pacientes, sobre todo para llegar a un consenso en cuanto al punto de corte que sería más útil para discriminar a unos pacientes de otros.

\section{BIBLIOGRAFÍA}

1. Tibble JA, Sigthorsson G, Foster R, Forgacs I, Bjarnason I. Use of surrogate markers of inflammation and Rome criteria to distinguish organic from nonorganic intestinal disease. Gastroenterol 2002; 123 (2): 450-60.

2. Suffredini AF, Fantuzzi G, Badolato R, Oppenheim JJ, O'Grady NP. New insights into the biology of the acute phase response. J Clin Immunol 1999;19 (4): 203-14.

3. Tibble JA, Bjarnason I. Non-invasive investigation of inflammatory bowel disease. World J Gastroenterol 2001; 7 (4): 460-5.

4. Fagerhol MK. Calprotectin, a faecal marker of organic gastrointestinal abnormality. Lancet 2000; 356 (9244): 1783-4.

5. Carroccio A, Iacono G, Cottone M, Di PL, Cartabellotta F, Cavataio F, et al. Diagnostic accuracy of fecal calprotectin assay in distinguishing organic causes of chronic diarrhea from irritable bowel syndrome: A prospective study in adults and children. Clin Chem 2003;49 (6 Pt 1): 861-7.

6. Canani RB, de Horatio LT, Terrin G, Romano MT, Miele E, Staiano A, et al. Combined use of noninvasive tests is useful in the initial diagnostic approach to a child with suspected inflammatory bowel disease. J Pediatr Gastroenterol Nutr 2006; 42 (1): 9-15.

7. Díaz Martín JJ, Codoceo Alquinta R, Sarriá Osés J, Molina Arias M, Prieto Bozano G, Polanco Allué I. Estudio de la utilidad de calprotectina fecal como marcador de inflamación en pacientes pediátricos con enfermedad inflamatoria intestinal. An Esp Pediatr 2002; 56 (Supl. 3): 25-6.

8. Roseth AG, Fagerhol MK, Aadland E, Schjonsby H. Assessment of the neutrophil dominating protein calprotectin in feces. A methodologic study. Scand J Gastroenterol 1992; 27 (9): 793-8.

9. Roseth AG, Schmidt PN, Fagerhol MK. Correlation between faecal excretion of indium-111-labelled granulocytes and calprotectin, a granulocyte marker protein, in patients with inflammatory bowel disease. Scand J Gastroenterol 1999; 34 (1): 50-4.

10. Tibble JA, Sigthorsson G, Bridger S, Fagerhol MK, Bjarnason I. Surrogate markers of intestinal inflammation are predictive of relapse in patients with inflammatory bowel disease. Gastroenterol 2000; 119 (1): 15-22.

11. Pardi DS, Sandborn WJ. Predicting relapse in patients with inflammatory bowel disease: what is the role of biomarkers? Gut 2005; 54 (3): 321-2.

12. Armas HM et al. Calprotectina fecal en el diagnóstico de la enfermedad inflamatoria intestinal. Comunicación Congreso. An Pediatr
(Barc) 2005; 62 (Supl. 2): 236.

13. Dale I, Brandtzaeg P, Fagerhol MK, Scott H. Distribution of a new myelomonocytic antigen (L1) in human peripheral blood leukocytes. Immunofluorescence and immunoperoxidase staining features in comparison with lysozyme and lactoferrin. Am J Clin Pathol 1985; 84 (1): 24-34.

14. Summerton CB, Longlands MG, Wiener K, Shreeve DR. Faecal calprotectin: A marker of inflammation throughout the intestinal tract. Eur J Gastroenterol Hepatol 2002; 14 (8): 841-5.

15. Johne B, Fagerhol MK, Lyberg T, Prydz H, Brandtzaeg P, Naess-Andresen $\mathrm{CF}$, et al. Functional and clinical aspects of the myelomonocyte protein calprotectin. Mol Pathol 1997; 50 (3): 113-23.

16. Hanai H, Takeuchi K, Iida T, Kashiwagi N, Saniabadi AR, Matsushita I, et al. Relationship between fecal calprotectin, intestinal inflammation, and peripheral blood neutrophils in patients with active ulcerative colitis. Dig Dis Sci 2004; 49 (9): 1438-43.

17. Fagerhol MK. Nomenclature for proteins: Is calprotectin a proper name for the elusive myelomonocytic protein? Clin Mol Pathol 1996; 49 (2): M74-M79.

18. Ton H, Brandsnes, Dale S, Holtlund J, Skuibina E, Schjonsby H, et al. Improved assay for fecal calprotectin. Clin Chim Acta 2000; 292 (12): 41-54.

19. Rasquin-Weber A, Hyman PE, Cucchiara S, Fleisher DR, Hyams JS, Milla PJ, et al. Childhood functional gastrointestinal disorders. Gut 1999; 45 (Supl. 2): II60-II68.

20. Gómez BJ, Ortiz C, Romero R, et al. Diagnostic yield of 335 push video-enteroscopies. Rev Esp Enferm Dig 2006; 98 (2): 82-92.

21. Bremner A, Roked S, Robinson R, Phillips I, Beattie M. Faecal calprotectin in children with chronic gastrointestinal symptoms. Acta Paediatr 2005; 94 (12): 1855-8.

22. Fagerberg UL, Loof L, Myrdal U, Hansson LO, Finkel Y. Colorectal inflammation is well predicted by fecal calprotectin in children with gastrointestinal symptoms. J Pediatr Gastroenterol Nutr 2005; 40 (4): 450-5.

23. García Sánchez MV, González R, Iglesias FE, Gómez CF, Casais JL, Cerezo RA, et al. Diagnostic value of fecal calprotectin in predicting an abnormal colonoscopy. Med Clin (Barc) 2006; 127 (2): 41-6.

24. Fagerberg UL, Loof L, Merzoug RD, Hansson LO, Finkel Y. Fecal calprotectin levels in healthy children studied with an improved assay. J Pediatr Gastroenterol Nutr 2003; 37 (4): 468-72.

25. Olafsdottir E, Aksnes L, Fluge G, Berstad A. Faecal calprotectin levels in infants with infantile colic, healthy infants, children with inflammatory bowel disease, children with recurrent abdominal pain and healthy children. Acta Paediatr 2002; 91 (1): 45-50.

26. Campeotto F, Butel MJ, Kalach N, Derrieux S, Ubert-Jacquin C, Barbot L, et al. High faecal calprotectin concentrations in newborn infants. Arch Dis Child Fetal Neonatal Ed 2004; 89 (4): F353F355.

27. Verdú Rico J, Juste M, Jover R, Muñoz Acosta J, Muñoz J, Martínez A, et al. La gammagrafía con leucocitis marcados con 99mTc-HM$\mathrm{PAO}$ en el diagnóstico y seguimiento de la enfermedad inflamatoria intestinal. An Pediatr (Barc) 2006; 64 (5): 457-63.

28. van der Sluijs Veer G, van der Horen B, Russel MG, van den Bergh FA. Time-resolved fluorimetric immunoassay of calprotectin: Technical and clinical aspects in diagnosis of inflammatory bowel diseases. Clin Chem Lab Med 2006; 44 (3): 292-8. 